ÉGYPTE monde arabe

\section{Égypte/Monde arabe}

$3 \mid 2000$

La censure ou comment la contourner

\title{
La Censure ou comment la contourner : introduction
}

Dire et ne pas dire dans l'Égypte contemporaine

Dyala Hamzah

\section{OpenEdition}

\section{Journals}

Édition électronique

URL : https://journals.openedition.org/ema/784

DOI : $10.4000 /$ ema.784

ISSN : 2090-7273

Éditeur

CEDEJ - Centre d'études et de documentation économiques juridiques et sociales

Édition imprimée

Date de publication : 30 juin 2000

Pagination : 11-23

ISBN : 2-87027-856-X

ISSN : $1110-5097$

\section{Référence électronique}

Dyala Hamzah, «La Censure ou comment la contourner : introduction», Égypte/Monde arabe [En ligne],

3 | 2000, mis en ligne le 08 juillet 2008, consulté le 07 juillet 2022. URL : http://

journals.openedition.org/ema/784; DOI : https://doi.org/10.4000/ema.784

Ce document a été généré automatiquement le 7 juillet 2022.

Tous droits réservés 


\title{
La Censure ou comment la contourner : introduction
}

\author{
Dire et ne pas dire dans l'Égypte contemporaine
}

\section{Dyala Hamzah}

1 Critique de cinéma, 'Alî Abû Shâdî devient censeur au ministère de la Culture avant d'être promu directeur de l'Organisme des palais de la culture. En sa nouvelle qualité, il fait publier, en janvier 2001, dans l'une des collections de l'Organisme ( littéraires»), trois nouveaux romans jugés "pornographiques» par un député récemment élu, affilié aux Frères musulmans. Indigné par cette littérature qu'il juge attentatoire à la pudeur, ce dernier soulève la question devant le parlement, entraînant la création, par le ministère de la Culture, d'une commission ad hoc chargée d'examiner les œuvres incriminées, laquelle recommande le retrait des œuvres de Yâsir Shacbân, Mahmûd Hâmid et Tawfîq 'Abd al-Rahmân. Craignant, s'il persiste, de prêter le flanc à des adversaires toujours prompts à l'accuser de gaspiller les deniers publics dans la publication de romans contraires aux valeurs morales et religieuses, le ministre cède aux injonctions de sa commission et remercie son serviteur. En signe de protestation et de solidarité, nombre de responsables démissionnent. «À censeur, censeur et demi » ou "Le censeur censuré », tel pourrait être le titre de ce mauvais scénario, n'était le fait qu'il ne s'agit guère ici de cinéma mais bien d'une affaire qui vient de défrayer la chronique dans le paysage politico-culturel égyptien. Tout en ayant un air de déjà-vu, cette affaire est exemplaire de ce qui apparaît comme une censure et une autocensure généralisées, d'autant plus pernicieuses qu'à aucun moment elles ne mettent en œuvre une décision de justice, seule à même de justifier, de légitimer, l'acte de censure. Car il n'est peut-être pas inutile de rappeler, au seuil de cette livraison, que la censure en Égypte possède un ancrage légal et que la liberté d'expression est non moins subjuguée par un syncrétisme juridique que par l'usage étatique et social, inconstant ou opaque, qui en est fait.

2 Si les articles 47 et 48 de la Constitution égyptienne garantissent la liberté d'opinion, de la presse, de l'édition et de l'impression et stipulent expressément l'interdiction de la censure sur la presse, une clause modificatoire ajoute que dans le cas où l'état 
d'urgence est décrété (voir loi $\mathrm{n}^{\circ} 62$ de 1958), une censure limitée "relative à des questions d'intérêt général » peut être exercée. Les articles 174 et 161 du Code pénal donnent un contenu tout général à ces questions d'intérêt général (la Constitution, l'ordre établi, les cultes), l'article 98 définit les peines encourues (prison, amendes), tandis que la loi $\mathrm{n}^{\circ} 20$ de 1936 identifie la personne juridique habilitée à saisir les imprimés en cas de conflit (le Conseil des ministres). Si les droits des auteurs sont codifiés par la loi $\mathrm{n}^{\circ} 345$ de 1954 et la recherche scientifique protégée par la Constitution (art. 49), la loi $\mathrm{n}^{\circ} 103$ de 1961 portant réforme d'al-Azhar donne à cette dernière un droit de regard sur les écrits. Par ailleurs, et contrairement au statut de l'imprimé (censurable après parution), la production théâtrale, cinématographique et vidéographique est soumise à une censure préalable (raqâba, dont les compétences relèvent du ministère de la Culture), ainsi que le prévoit la loi $n^{\circ} 430$ de 1955 . Une circulaire de 1947 n'énonce à cet égard pas moins de 33 interdictions dans les domaines moral et social et 31 dans les domaines de la sécurité et de l'ordre public'. Enfin, tout un arsenal de lois et de décrets relatifs à la protection des "secrets d'État " (loi n 100 de 1971), des informations militaires (loi $n^{\circ} 14$ de 1967) ainsi qu'à la conservation et la publication des archives de l'État (loi $n^{\circ} 121$ de 1975 et décret $n^{\circ} 472$ de 1979) vient couronner l'ensemble d'un dispositif, servi par une police des œuvres artistiques (ministère de l'Intérieur) et de plus en plus fréquemment épinglé par la Haute Cour constitutionnelle égyptienne' comme étant anti-constitutionnel.

3 La protection en principe de la liberté de création par la loi fondamentale est en fait soumise à des restrictions correspondant au statut des intellectuels toujours défini par le nassérisme (rôle « constructif » de l'intellectuel au service de l'idéologie nationale); par voie de conséquence, au problème de la représentation de l'Égypte (comment peutelle et doit-elle être représentée à l'extérieur, qui a le droit, et dans quel but, de s'approprier la mémoire et les institutions de cet État); au rôle dévolu à l'islam officiel ("nationalisation» d'al-Azhar à qui on concède juridiquement une autorité dogmatique et morale contre la tâche de traduire en termes "islamiques » l'idéologie régnante) ; enfin, au régime de l'état d'urgence, encore en vigueur aujourd'hui'. Quant au recours qu'on en a, il ne semble pas nettement ressortir à un principe intangible. Pourquoi en effet Hamdî Batrân a-t-il été condamné par une juridiction militaire pour ses Yawmiyyât zâbit bi-l-aryâf alors qu'Infijâr jumjuma de Ismâ̂îl 'Alî, où l'armée en prend pour son grade de bout en bout, n'a pas été interdit? Comment expliquer que Sonallah Ibrâhîm n'ait jamais même été inquiété alors qu'un interdit pèse encore sur la publication de Awlâd hâratna du prix Nobel Naguib Mahfouz? Ou que l'instance administrative ait pu entraver un temps la décision judiciaire en faveur du film alMuhâgir de Youssef Chahine? La politique du cheveu de Mucâwiya ne saurait seule expliquer les initiatives d'al-Azhar, du parquet, du ministère, et les décisions judiciaires et administratives subséquentes. S'il ne faut sans doute pas négliger les personnes derrière les institutions', c'est plutôt ou conjointement dans des stratégies et des techniques discursives qu'il faudrait chercher la réponse, et qui permettraient de comprendre à la fois comment la censure est pratiquée, intériorisée et contournée.

4 Mais l'arsenal juridique, son interprétation héritière et ses inflexions circonstancielles ne président pas seuls aux destinées du dire. Un puissant contrôle social est aussi assuré par des initiatives individuelles qui cherchent pour leur croisade morale et/ou religieuse, une traduction et une codification juridiques (ainsi des récentes requêtes en hisba, ou préservation de l'ordre islamique'); des voies de fait, qui vont de la menace et 
de l'intimidation à l'assassinat (Farag Foda) ou aux tentatives d'assassinat (Naguib Mahfouz). Témoignage de ce qu'il n'y a pas de véritable monopole institutionnel de la morale et/ou de la religion, ce contrôle atomisé, affecté de pratiques agies et informées par ce référent, triomphe également dans une autocensure multiforme, qui peut prendre, chez les auteurs, la forme extrême du dédit, chez les éditeurs, d'un retrait de la vente, chez les uns et les autres, la recherche ou le passage par d'autres publics, d'autres marchés.

5 Cette présentation des parties prenantes à la censure ne devrait pas faire accroire que la logique des intérêts en jeu se traduit aisément, pour ne pas dire schématiquement, en termes d'opposition entre nationalisme et islam. L'enchevêtrement constant de ces deux référents doit être postulé comme rationalité à l'œuvre dans la censure si tant est que l'on veuille débrouiller l'écheveau d'inintelligibilité qui semble caractériser ces affaires. Deux points peuvent ici servir d'illustration à cette imbrication des référents : le fait que les présentatrices de télévision ne peuvent jamais être des femmes voilées en même temps qu'elles ne doivent pas apparaître avec le moindre décolleté; le fait qu'une loi censée circonscrire les menaces contre la sécurité nationale, ait pu, à l'ère sadatienne, être édictée en termes aussi ambigus que ceux de qânûn al-cayb ou loi de la honte...

6 Cette situation n'est pas neuve mais une conjonction de facteurs socio-politiques nouveaux et anciens se combinent désormais pour rendre ses enjeux plus saillants. Sur la scène intérieure, si l'État commence à avoir raison de la lutte islamiste armée (la répression militaire et judiciaire n'est sans doute pas pour rien dans les récents appels des fondateurs des organisations armées - Jihâd, Jamâca islâmiyya - à l'arrêt de la violence), s'il commence également à resserrer son étau autour des mosquées, souvent lieu de dissémination de la parole islamiste (le ministère des Waqf faisait connaître en juin 1998 son plan de mise sous tutelle étatique, d'ici l'an 2000, des 65000 mosquées du pays), la surenchère idéologique de cette dernière trouve toujours des relais efficaces à l'université (affaires "Nasr Abû Zayd", "Samia Mehrez », "Didier Monciaud») et montre ainsi que sa pénétration sociale a encore de beaux jours devant elle. D'autant que l'État s'est résolument engagé sur la voie de l'ajustement structurel, facteur de fragilisation et de paupérisation d'une société de laquelle il s'est tout aussi résolument désengagé ; et que les effets de la globalisation ne font qu'aggraver des inégalités et des déséquilibres sociaux autrement visibles. Cela étant dit, et même si à cette surenchère islamiste peut correspondre une anticipation étatique ou sociale (cette dernière pouvant prendre la forme de la conformisation ou de l'initiative), le temps n'est plus aux concessions politiques majeures : la loi n 3 de 1996 sur la hisba abolit virtuellement du droit égyptien les recours en préservation de l'ordre islamique.

7 Sur la scène extérieure, les accusations de plus en plus fréquentes, d'autant plus insistantes qu'elles sont précises, par les ONG, de violations des droits de l'homme en général, en particulier du droit à la liberté d'expression, sont désormais d'autant plus embarrassantes et inévitables qu'elles sont relayées par des instances gouvernementales, ou des organes de l'opinion publique occidentale. Ainsi de ces listes dressées des œuvres interdites en Égypte ces dernières années, publiées par des ONG locales, reproduites dans tel grand quotidien américain. Ainsi de la désignation par des magazines américains, anglais, français du "problème confessionnel» de l'Égypte, cheval de bataille des "Coptes de l'étranger " et de l'inscription de l'Égypte, par le Sénat américain, sur la liste des pays où sévit la persécution religieuse. Comme si, 
désormais, les gages donnés à l'Occident (soutien inconditionnel aux alliés pendant la guerre du Golfe, engagement indéfectible dans le processus de paix israélo-arabe) ne suffisaient plus pour obtenir un blanc-seing en matière de libertés prises avec la liberté d'expression.

Pour ne pas être neuve, la situation n'est pas spécifique non plus. Nonobstant ses applications nassériennes, ce n'est pas sur les rives du Nil que le maccarthysme, dans ses versions institutionnelle et non institutionnelle, est né, et l'interdiction de diffusion en Égypte (comme ailleurs dans le monde arabe) de la réédition londonienne d'al-Rawd al-câtir, ce traité d'érotisme du XVe siècle de cheikh al-Nifzâwî, vendu depuis toujours trois francs six sous dans tous les souks du Maghreb, n'a rien à envier à l'interdiction de vitrine qui frappe Sade en France jusque dans les années soixante-dix: dire dans la pénombre des arrière-boutiques et dans le cercle restreint de " ceux qui savent », oui ; dire au grand jour publicitaire, non. C'est en France aussi que jusque dans les années cinquante on intente des procès littéraires contre des œuvres jugées attentatoires aux bonnes mœurs. Ni sorcière de Salem ni Dame autodafé, la censure (officielle) à l'égyptienne cependant, à force de ménager la chèvre et le chou, finit par naviguer en eaux troubles : si les acteurs d'aujourd'hui " peuvent identifier ce que fut «la longue marche » de leurs aînés, tour à tour pris aux pièges de la censure anglaise, de la censure monarchique, ou celle de l'État nassérien; s'ils ont appris, en lisant les mémoires de leurs historiens, à anticiper sur l'interdiction pour mieux en déjouer les pièges et observé, dans l'expectative, l'ouverture sadatienne vite refermée", qu'en est-il aujourd'hui ? où mène, en effet, ce brouillage de ses stratégies (frapper aussi quand on s'y attend le moins et vice versa), vécu par les acteurs comme une perte de repères idéologiques (frapper au nom de l'islam bien compris? du credo nationaliste ?...) et une inintelligibilité des mobiles (maintenir coûte que coûte une "solidarité sans consensus $\|^{\prime}$ ) ? Dans cette incertitude des alliances et dans ce manque de visibilité des institutions de l'État et de la société naissent pourtant, paradoxalement en retour, des stratégies de contournement et de résistance, des modalités de l'énonciation, une inventivité du dire, affinées, aiguisées, par l'autre censure, celle diffuse et noninstitutionnelle.

Quand dire c' est faire ou les domaines d'investigation

$\mathrm{Ni}$ bilan des pratiques répressives et de leurs conséquences sur la production intellectuelle et artistique en Égypte (on se reportera pour cela à l'ouvrage de Fathî Ghânim, al-Maraka bayn al-dawla wa-l-muthaqqafin), ni histoire de la censure au sens large et de ses modalités, cette livraison s'est voulue toute entière à l'écoute de ce qui se dit et de ce qui ne se dit pas' aujourd'hui en Égypte, c'est-à-dire, de ce qui se fait : acte qui engage dans l'espace public, posé sur l'horizon d'une réalité perçue dans sa massive naturalité ou comme construit social' (c'est ce que l'approche par les stratégies des acteurs seule pourra déterminer), le dire est un faire, non seulement en son sens strictement performatif et austinien ${ }^{10}$ mais en ceci qu'il est indissociable de la contextualité dans laquelle il advient, de l'instauration d'un avant et d'un après, et qu'il est une production et une modification (de la langue; de la relation et de sa dynamique). Cette double approche par l'ethnométhodologie (Sharrock et Watson") et l'anthropologie culturelle (de Certeau") a l'avantage de poser l'unité de l'agir (ainsi que l'inconfort de ses apories!), en même temps qu'elle permet de retenir au-delà du seul écrit, toute activité discursive non imprimée. Presse, cinéma et littérature (savante ou romanesque) sont ici nos seuls domaines d'investigation: si pour de Certeau, l'activité 
scripturaire est fondatrice de la modernité, nous poserons que l'enregistré sous toutes ses formes est le lieu par excellence du dire dans son unité avec le faire. Romans, essais, presse écrite mais aussi audio-visuel, théâtre et cinéma appartiennent de plain-pied à cette modernité en ce sens qu'ils participent de cette vaste entreprise d'annexion rationnelle du monde et ils appartiennent au faire, en ce sens qu'ils produisent ou modifient un réel posé comme un fonds inépuisable de ressources.

Pourquoi ces choix exclusifs de l'oralité qui, chez de Certaux encore, comprend la parole de la folie, de l'enfance, du peuple? Ne risquait-on pas, par cette exclusion, de réinstaurer une scission entre le dire et le faire? L'oralité n'est-elle pas également un faire et un faire qu'on peut enregistrer et transcrire (la psychanalyse, la psychologie, l'ethnologie, le folklore en témoignent) ? Certes, mais à cette nuance près que cette parole-faire n'a ni vocation universelle ni ne coïncide avec son support scripté. En cela, elle est l'autre radical du dire auto-enregistré : son éventuel enregistrement ne l'épuise jamais puisque ce qu'elle dit n'a pas vocation à être traduite en même temps que dans cette médiatisation, elle cesse d'être un faire. Le discours du fou (ou de l'analysant), la parole de l'enfant, la chanson populaire ne sont des faire que pour autant qu'ils coïncident avec l'oralité. Ce n'est pas le cas de l'auto-enregistré qui est un faire parce qu'il est ou en ce qu'il est auto-enregistré. Cessant d'être un faire dans sa médiatisation, l'oralité ne donne donc plus aucune prise à la censure et sort de fait de notre domaine d'investigation. Ce détour par les analyses lumineuses de l'auteur de L'invention $d u$ quotidien aura aussi eu pour vertu de rendre problématique le poncif selon lequel l'oralité représente ce chemin de traverse qu'emprunterait l'expression autrement muselée : l'oralité n'est pas un mode de l'autocensure, une stratégie de contournement de l'interdit. L'oralité est cet autre radical de l'écrit (ou de l'auto-enregistré) précisément en ce sens qu'elle se soutient elle-même : sa transcription ou traduction ultérieure ne lui ajoute aucune dignité, pour ne pas dire qu'elle l'altère. Ainsi ce qui se dit dans la nukta ne peut se dire ailleurs ou autrement que dans la nukta. La nukta représente donc, à part entière, une espèce (de parole-faire) d'un genre (de parole populaire) : elle n'est l'ersatz d'aucune parole, ni d'aucun genre « scriptible ».

11 La folie, l'enfance, le peuple : dans d'autres développements, de Certeau rajoutait la parole religieuse dans ses délimitations des lieux de l'oralité. Non qu'il ait eu en tête la parole religieuse comme dogme et comme Écriture, bien sûr, mais bien cette parole soutenue par l'activité rhétorique (rhétorique dont il dit si bien qu'elle fut la première victime de la modernité scripturaire !). En toute logique, il aurait donc fallu inclure ici l'orateur politique: prêches et discours, en ce qu'ils se soutiennent des effets de l'oralité charismatique et de l'activité du tribun, s'ils trouvent mille modalités d'enregistrement, d'amplification et de diffusion, restent avant tout des paroles-faire et, nonobstant la démultiplication de l'auditoire par ces moyens de diffusion visant à l'universalisation de cette parole, ces «dires" restent non pas simplement entachés d'oralité, mais tout entier investis par cette dernière. Inversement, le dire qui nous a interessé n'est ni la traduction/transcription d'une oralité, ni son soutien ou son substitut. De même qu'il est vain de rechercher dans "l'adaptation" cinématographique d'un livre, une fidélité à sa raison et à sa cohérence (à son message !?), de même serait-il futile d'attendre de la raison scripturaire qu'elle puisse se « dire » oralement. Comment on investit aujourd'hui les genres constitués du dire écrit et enregistré, voilà ce qui seul nous a occupé ici.

Entre les mailles du filet : façons de faire 
Les deux contributions qui ouvrent ce dossier fournissent des clés pour l'intelligibilité et la contextualisation du fonctionnement de la censure au double niveau juridique et anthropologique. Ahmad Sayf al-Islâm Hamad ( L'intervention administrative dans la liberté d'expression. Entre loi et Constitution ») montre ici le louvoiement législatif et la latitude interprétative que se permet le régime dès lors qu'il s'agit d'accompagner la mise en pratique de certaines dispositions constitutionnelles, en l'occurrence, ici, la liberté d'expression. Les leviers de l'ingérence administrative dans la liberté d'expression que représentent les lois sur les imprimés et sur l'état d'urgence apparaissent ici dans toute leur inconstitutionnalité lorsque l'auteur rappelle la jurisprudence de la Haute Cour constitutionnelle en la matière. Ce qui est souligné en creux, c'est une certaine schizophrénie du politique, le régime ne se confondant pas avec l'État sur la question de la définition des rapports entre libertés et ordre public, et c'est dans l'espace contradictoire inauguré par cette scission que s'engouffre et tente de se maintenir la liberté d'expression, muselée tant bien que mal au gré des rapports de force institutionnels. Mais l'analyse par les structures, comme d'ailleurs par les stratégies d'acteurs, ne peuvent seules suffire dès lors qu'il s'agit de comprendre comment et pourquoi la censure paraît normale au point de ne jamais engager de débats sur sa nécessité (sauf, peut-être, celle qui concerne la censure politique) : JeanNoël Ferrié («Censure et sens commun en Égypte ou l'invention répétée de la normalité islamique ») entreprend ici d'établir ce point en déportant l'analyse dans les opérations de catégorisation cognitive de l'anthropologie. Cette normalité s'explique-t-elle par une identification de la censure à une défense et illustration de l'islam, puisqu'il est entendu que tout le monde est pour un islam quelconque, quelque soit le contenu que l'on donne à ce dernier? Non, et J.-N. Ferrié montre clairement que la référence à l'islam dans les discours n'ajoute rien aux opérations de catégorisation, mais qu'elle ressortit à ce que Jon Elster nomme «l'obsession du sens ». Ce qui expliquerait bien plutôt cette «naturalisation » de la censure, c'est son identification au sens commun. $\mathrm{Au}$ delà de sa vertu fondatrice, cette identification a par ailleurs le mérite d'unifier sous un même vocable ce que les contributeurs de cette livraison nomment tantôt « censure de la rue ", tantôt " censure informelle », tantôt encore « censure populaire ».

Les cinq articles qui suivent illustrent, chacun dans un domaine particulier (fiction, exégèse, cinéma et presse) et en faisant la part belle à l'empirie, les stratégies déployés par les acteurs sur ce double horizon censorial. En balayant le champ littéraire égyptien contemporain, Richard Jacquemond («Les limites mouvantes du dicible dans la fiction égyptienne ") avance deux raisons à la recrudescence paradoxale des affaires de censure dans le contexte libéral de la décennie écoulée : le retour au modèle nassérien de contrôle des intellectuels et une "poussée révolutionnaire » dans le milieu de la fiction, où une nouvelle génération fait accéder à la dignité littéraire des pans entiers de l'individu et de la société, jusque-là occultés. Ce faisant, il avance un sens au recours a priori anarchique à la censure. Pour schématiser son propos, la fiction égyptienne peut s'attaquer à ce qu'elle identifie comme tabou, pour autant qu'elle le fait en endossant et en assumant le rôle du Bildungsroman, mais d'un roman d'éducation revisité par le projet nationaliste. Ainsi, c'est le paradigme « réaliste-réformiste » qui explique non seulement les destins éditoriaux différents d'œuvres à la thématique en apparence similaire mais, in fine, l'échec de la scène littéraire égyptienne à fonder (si tant est qu'elle existe ailleurs!) une conception autonome de l'art. Anwar Moghith ( " Le texte et la marge : critique des mythes de l'Ancien textament chez 'Isâm al-Dîn 
Hifnî Nâsif ») fait, quant à lui, parler une certaine critique marxiste égyptienne de la religion et montre que si la déconstruction du judaïsme peut être frontale (grâce à l'exploitation politique qu'on peut en faire), l'implicite triomphe dès lors qu'il s'agit de s'attaquer à l'islam. L'instrumentalisation d'une tradition scripturaire dans la critique d'une autre tradition scripturaire, et, pour ce faire, la mise en place d'une véritable technique de la juxtaposition et de la suggestion, font ici de Nâsif un virtuose du nondit. Du non-dit au ne-pas-dire, il y a un pas qu'un certain cinéma s'est empressé de franchir afin de tenir le haut de l'affiche : en s'intéressant au cinéma grand public des années quatre-vingt-dix du point de vue des acteurs sociaux eux-mêmes, Dina Galal ("Le cinéma grand public égyptien entre censure et guichet») montre comment l'intériorisation de la censure se confond ici avec son contournement, ainsi qu'en témoigne l'apparition de ce cinéma modelé par les conditions de sa réception. En s'attardant sur les personnes des censeurs et en s'attachant à les faire parler, elle éclaire d'un jour nouveau un aspect galvaudé de la censure - celui relatif à l'origine socioprofessionnelle des censeurs, souvent issus des milieux artistiques, et ex-victimes potentielles ou effectives de la censure : l'intériorisation institutionnelle de la nécessité censoriale est ici telle qu'on met autant d'application à s'acquitter de sa mission de créateur-victime (forcément victime, car il y a aussi une mission du créateur-victime, celle consistant à éduquer la censure !) qu'à s'acquitter de celle de redresseur de tort idéologico-esthétique ; telle, surtout, que l'on passe de l'une à l'autre vocation avec une égale détermination, une autojustification et un sens du devoir que ne semble jamais remettre en question cette nécessité même de la censure, évoquée tantôt par J.-N. Ferrié. Une intériorisation non pas tant économique que politique de cette dernière est par suite illustrée par Nathalie Bernard-Maugiron et Gamal Abdel Nasser Ibrahim (« Pouvoir de la censure ou censure du pouvoir? L'affaire Yûsuf Wâlî contre al-Shacb») qui montrent comment des journalistes instrumentalisent la censure à des fins politiques. Pour les chroniqueurs d'al-Shacb - mais cela est aussi vrai d'autres journaux, d'autres journalistes -, les procès en diffamation intentés contre eux par les figures publiques épinglées dans leurs colonnes représentent le moyen le plus sûr d'accéder à une parole politique autrement muselée. C'est ainsi que les tribunaux deviennent, paradoxalement, de véritables tribunes libérant, à défaut de plateformes et de programmes, une critique du régime. Si leurs chevaux de bataille journalistiques sont l'immoralité publique et la corruption, mesurées l'une, à l'aune d'une islamité bien entendue, l'autre, à celle des principes de la gouvernance (accountability, transparence, etc.), leur mise en examen figure ici un «militantisme politique » d'un genre nouveau : en prenant le risque d'aliéner leur liberté individuelle, ils signifient en creux que la simple interpellation citoyenne de responsables non comptables est sinon impossible du moins à ce prix aujourd'hui en Égypte. En s'intéressant à ce qu'elle nomme le nouveau journalisme égyptien, Dina El Khawaga («Sisyphe ou les avatars du nouveau journalisme égyptien ») montre la stratégie acharnée d'une certaine presse qui fait feu de tout bois pour exister et survivre dans le paysage sclérosé de la presse égyptienne : de la sollicitation d'une licence de presse privée à l'investissement d'une licence de presse partisane en passant par l'obtention d'une licence étrangère et la recherche de prête-nom, le journal al-Dustûr et son rédacteur en chef Ibrâhîm cîsâ n'ont eu de cesse de répondre à la surenchère censoriale par une surenchère de la résistance et de la persévérance. Ce, jusqu'aux confins d'une autocensure tragicomique, comme en témoigne l'embauche par la rédaction d'un ex-censeur, chargé d'opérer, sur le « bon-à- 
tirer ", une censure blanche afin de permettre au journal de passer l'épreuve à laquelle est soumise la presse étrangère.

Enfin, la présentation magistralement documentée, par Mustapha al-Ahnaf, de "L'affaire Haydar Haydar", illustre non seulement le mécanisme par lequel une « affaire » est fabriquée de toute pièce (comment elle descend dans la rue et monte à la une des journaux) mais aussi comment elle conforte les représentants de chaque bloc dans la stérilité de leurs ressassements. Ce faisant, elle démontre comment le régime instrumentalise ces mêmes affaires à ses propres fins, aux fins de son auto-maintien. En retirant du marché l'ouvrage "athée " publié par ses propres institutions, en suspendant le journal par lequel le scandale est arrivé et en interdisant le parti dont il est l'organe, le régime fait plus que sauver sa face. Il s'affirme à la fois fidèle à l'islam et au nationalisme, coupant l'herbe sous le pied à la critique des deux bords, par cette opération de maintien ou de retour à l'ordre.

La difficulté de nommer ou la typification de l'interdit

Définir les modalités du dire dans un contexte de censure supposait les objets de la censure clairement identifiables, ce qui n'allait pas de soi. Certes, des textes sont là qui donnent un contenu positif à l'interdit (voir la circulaire de 1947 pour le théâtre et le cinéma, déjà citée), mais on l'a vu aussi, des pratiques sont là qui font mentir ces textes, au mieux, les décrédibilisent ('Alî vs. Batrân). Du texte à la pratique, y a-t-il eu, au fil du temps, des brèches ouvertes dans la trilogie interdite (sexe, politique et religion), exemplifiée par le Syrien Abû 'Alî Yasîn dans son al-Thalûth al-muharram? En s'intéressant aux affaires récentes de censure universitaire (rubrique "En-Notes"), Mustapha al-Ahnaf propose bien de rajouter à cette trilogie consacrée un quatrième volet (celui de l'obscénité). Si donc, pour prendre un exemple, un rapport Kinsey sur la sexualité des Égyptiens est aujourd'hui plus impensable que jamais, a fortiori n'est-il pas question de parler de brêches mais plutôt de prédications restrictives et évolutives de ce qui, en religion, en matière de sexe et en politique, est interdit. À travers la recension du censuré, il devenait alors possible de typifier l'interdit - typification d'autant plus nécessaire qu'elle représente la condition d'une intériorisation réussie de la censure. Plus l'interdit parait brouillé, moins l'autocensure opère à travers ses tabous structurants. Ainsi de la triple stratification identitaire de l'Égypte (égyptianité, arabité, islamité), de la question de la normalisation culturelle avec Israël (tatbîc), du rapport à la langue, à certaines coutumes, à la question de la citoyenneté, à celle de la foi... Ces contenus positifs de l'interdit ne représentent pas encore des types de l'interdit mais servent à les nourrir : ces catégories générales peuvent prendre pour noms "rapport à soi ", "rapport à l'autre " et "rapport au monde». Elles peuvent signaler par suite et les types de déviances et les types de déviants, marginalisés dans l'acte même de la désignation: le traître, l'athée/apostat, le criminel et le fou. Ces catégories générales de la réprobation universelle ne sont par suite spécifiques et spécifiées ici que dans l'acte même de la censure qui les consacre et les informe en même temps. On n'est pas traître, athée ou criminel en France comme on est traitre, athée ou criminel en Égypte: encenser le dialectal et lui donner une dignité linguistique (par la codification, son usage en littérature,...), montrer l'excision, remettre en question le triptyque identitaire, avoir une approche seulement civilisationnelle ou culturelle de l'islam, voilà de quoi se mettre de facto au ban de la communauté. 

paradoxalement possible de dire, c'est-à-dire d'inventer ces stratégies discursives, qui doivent sans doute autant au capital social et à l'imagination individuels qu'aux legs de savoir-faire hérités des aînés. Un bornage naturel des lieux du dit et du non-dit consisterait à circonscrire l'investigation dans les limites extrêmes représentées par la langue de bois et la transgression. Ce n'est pas à dire qu'au sein de cet espace il faille recourir à un balayage exhaustif, aussi chimérique d'ailleurs que non-pertinent. Bien plutôt, le dit et le non-dit se traquent ici :

1) dans ce qui se dit autour des œuvres censurées ou des affaires de censure (Jacquemond, al-Ahnaf) : dans cette catégorie, la puissance et le triomphe de la censure se mesurent à l'aune de l'orientation que prennent les débats. Cette puissance et ce triomphe ne sont jamais aussi grands que quand ils semblent épuiser ces derniers en termes de " pour » et de " contre ». Dès lors, l'œuvre incriminée n'est lue ou vue qu'à travers les seuls critères de la censure, posés comme valides. Pourtant, à travers cette polarisation, l'instrumentalisation de l'objet censuré sert aussi les desseins d'une critique du régime (lorsqu'on se déclare contre la censure) tandis que l'instrumentalisation des arguments de la défense sert les objectifs d'une critique d'un mode d'être intellectuel et savant (lorsqu'on fustige l'autocensure). Enfin, les objectifs ponctuels de la censure (interdire une pensée libertine ou hérétique) sont de toutes façons toujours subsumés sous une rationalité qui échappe en partie à ses acteurs et qui échappe de toutes les manières tant qu'on reste le nez dessus. Ce qui par exemple se donne à lire dans les polémiques et interdictions diverses qui frappent la réédition des Mille et une nuits (dans sa version non expurgée de la fin des années 70) et celle des Futuhât makkiyya d'Ibn 'Arabî (commencée dans les années 80, suspendue, reprise et à nouveau suspendue avec la mort de 'Uthmân Yahyâ), au-delà du superficiel «ce n'est pas de la littérature ", c'est bien une exclusion de ces œuvres d'un patrimoine que seules seraient autorisées à habiter des œuvres jugées « islamiques » du point de vue de la croyance et de son affirmation et non du point de vue de la civilisation.

2) dans ce que disent les œuvres heureuses (Galal) et paradoxalement heureuses (Moghith), celles réunissant tous les «ingrédients» de la censure et qui pourtant ne sont pas censurées : ici se pose la question du pourquoi (ces silences ou ces ratés de la censure) à laquelle on tâchera de répondre en posant la question du comment (ces auteurs heureux font-ils pour échapper au couperet). L'approche par la sociologie de la connaissance est ici féconde, surtout celle, spécifique, de Léo Strauss, qui, dans La persécution et l'art d'écrire", a si bien montré les techniques d'écriture entre les lignes déployés par les auteurs poursuivis ou mis à l'index: la technique particulière de superposition de bribes de récits de vie et de coupures de presse dans le roman Dhât de S. Ibrâhîm peut-elle expliquer la déroute de la censure, par ailleurs pleine d'assurance dans le cas d'un auteur internationalement consacré ?

19 3) enfin, dans ce que donnent à entendre, sur le mode du «listening between the lines " $"$ des polémiques et des débats nationaux récurrents (voir, par exemple, celui sur la réforme récente de la loi sur les associations et les organisations non gouvernementales), des réactions à des événements extérieurs ou intérieurs (Louxor : consensus sur la thèse du complot...), à des phénomènes de société (l'affaire des « adorateurs de Satan »; les évanouissement collectifs dans les lycées du gouvernorat de Buhayra ; les récents cas de mariages curfî parmi la jeunesse universitaire). Comment s'empare-t-on d'un objet particulier, comment l'investit-on pour parler d'autre chose?

Égypte/Monde arabe, 3 | 2000 
Les débats animés par Buthayna Kâmil dans son émission radiophonique I‘tirâfât layliyya, ainsi que dans sa rubrique du même nom dans feu al-Dustûr tout comme les campagnes diffamatoires des journalistes d'al-Shacb jettent une lumière sur un investissement que l'on pourrait qualifier de métonymique. Parler de la polygamie, de l'inceste ou de l'adultère alors qu'on s'attaque à des problèmes sociaux qui les dépassent et les subsument; dénoncer la corruption et la normalisation pour remettre en cause non pas une politique mais la légitimité tout entière d'un régime sont des exemples parmi une pléthore d'autres. C'est bien cet investissement métonymique par le sensationnalisme ou par la diffamation qu'on a sanctionné en censurant al-Dustûr (El Khawaga) et al-Shacb (Bernard-Maugiron \& Ibrahim)

Géographie du dire

Si le modèle de la langue a pu convenir pour analyser ces "stratégies", ces " techniques ", c'est parce qu'il pose un corpus de règles dans son rapport à ses usages. Et c'est bien ici dans l'écart par rapport à une grammaire intériorisée que la parole traquée advient quand même. Saussure, le premier, en posant la langue comme système et la parole comme inventivité": Chomsky, en distinguant la "compétence » de la "performance " linguistiques", font figures de pionnier en même temps que leur cantonnement dans la générativité des règles ne peut ici nous servir dans notre recherche de la générativité (de la généalogie) des usages. Wittgenstein (« don’t ask for meaning, ask for usage »), de Certeau (l'» usage » contre la consommation passive) sont bien plutôt pertinents pour nous ici dans la mesure de leur insistance sur l'écart et le déplacement (aboutis, réussis !) par rapport à une norme. À partir de là, quelques trois axes stratégiques ont émergé sur lesquels il nous a semblé voir se déployer la géographie du dit et du non-dit, géographie qui se déploie non seulement sur l'horizon de la parole interdite mais conjointement sur celui d'une parole autorisée, celle, par exemple, de la « culture vulgaire » (al-thaqâfa al-hâbita):

21 A) la stratégie qui consiste à investir le même, l'identité dans l'infime écart, ou comment réinvestir de vieux paradigmes, des credos sacrés, des axiomes consacrés". Ce sont ici les entreprises heureuses de cette littérature "réformiste-réaliste " (Jacquemond); de ce cinéma "grand public ", qui fait mine de s'attaquer à la trilogie tabou alors qu'il sert des objectifs politiques (Galal); d'une critique de l'islam qui se sait en terrain sûr dès lors qu'elle revêt les oripeaux d'une critique du judaïsme en s'inscrivant dans cette tradition exégétique hostile aux apports bibliques (Moghith).

B) La stratégie de la volte-face, de l'altérité, qui consiste à brouiller les cartes en investissant un autre domaine d'expression ou un autre média. Ainsi de ces anciens militants de gauche qui investissent désormais le champ littéraire. L'activisme politique est verrouillé, mais la critique littéraire offre des possibles pour l'expression. Les petites revues avant-gardistes (al-Kitâba al-ukhra, al-Garâd) sont tout à fait représentatives de cette "déportation » du faire (Jaquemond). Ainsi de ces journalistes qui n'écrivent plus sur le mode diffamatoire que pour investir les tribunaux, qui cherchent, à travers une judiciarisation du dire, un faire politique (Bernard-Maugiron \& Ibrahim).

C) La stratégie de l'ubiquité (être ici et là-bas) qui consiste à la fois à se conformer et à transgresser, et à transgresser d'autant mieux qu'on adopte, ailleurs, le rôle de l'»entrepreneur de morale »". Cette maîtrise, qui suppose bien une certaine " compétence politique », doit être prise au sérieux comme telle puisqu'elle est à la fois moins et plus qu'une simple duplicité, qu'un effet de génération, que le signe d'une 
évolution. Ce que signale l'au-delà de ce double langage, cette schizophrénie qui signe l'impossible présence à soi-même, c'est sans doute en fait l'impossible présence effective des acteurs à leur présent. Si la transparence est sans prise sur le réel, si l'entièreté donne trop de prise à ce dernier, la conjonction des deux est une façon d'exister ici tout en étant là-bas. C'est peut-être dans ce cadre qu'il faut comprendre comment Gamâl al-Ghîtânî, que l'histoire retiendra comme l'un des pionniers du renouvellement de la littérature arabe, a pu à la fois signer al-Zaynî barakât et se poser en pourfendeur acharné d'al-Saqqâr (Jacquemond). C'est très certainement dans cette optique qu'il faut appréhender l'originalité transgressive d'al-Dustûr, et le conservatisme populiste d'I. cÎsâ dans l'affaire Haydar Haydar (El Khawaga ; al-Ahnaf) ; ou encore, la dualité surprenante de M. cAbbâs, initiateur de cette même affaire Haydar Haydar et lui-même auteur de romans qui lui vaudraient les mêmes désagréments que sa victime si d'aventure on s'amusait, entre autres, à en citer des passages hors de leur contexte...

Faut-il dire, pour clore, que cette géographie advenue du dire est le symptôme d'un malheur politique relatif? La tentation est bien sûr grande de vouloir comparer. Mais les accommodements et les techniques ne peuvent jamais faire oublier qu'ils se pratiquent dans la connaissance de l'économie des équilibres, dont le principe est l'état d'urgence. Peu de temps après sa libération provisoire, en août 2000, Sacad al-Dîn Ibrâhîm, professeur de sociologie et fondateur du Centre Ibn Khaldûn pour le développement, annonçait, dans une conférence donnée à l'université américaine du Caire, deux bonnes nouvelles relatives à sa détention estivale : primo, les services de la Sûreté de l'État avaient bien voulu attendre le matin pour l'appréhender, rompant avec une longue et sinistre tradition d'arrestations nocturnes; secundo, il n'avait pas été torturé. Si l'humour grinçant de la première annonce témoignait de ce que l'intégrité physique et mentale de l'homme n'avait pas été atteinte, la seconde annonce, qui confirmait ce fait, signalait par sa sécheresse et une angoisse à peine contenue du ton, la persistance d'une pratique nettement moins comique, qui épargne peut-être désormais les figures connues de la "société civile", mais continue de s'appliquer à d'autre. C'est cette incapacité du régime à tolérer et à incorporer ces "autres", les islamistes pour ne pas les nommer, qui signe la limite absolue du dire en Égypte. Le maintien dans l'illégalité des Frères musulmans et, récemment, la suspension du Parti du Travail et de son organe alshacb", témoigne de ce que cette intolérance, loin de s'adoucir, connaît au contraire un regain de mauvais augure.

\section{NOTES}

1.Bernard Botiveau, «Penser, dire et interdire : logiques et enjeux de la censure des écrits en Égypte ", Égypte/Monde arabe, n 14, 2 trim. 1993.

2.Au sujet de la montée en puissance de cette juridiction, de son opposition de plus en plus fréquente aux lois promulguées hâtivement par un État qui, jusque-là, avait les coudées franches, voir la précédente livraison d'Égypte/Monde arabe $\left(\mathrm{n}^{\circ} 2:\right.$ : Le Prince et 
son juge : Droit et politique dans l'Égypte contemporaine »), tout entière consacrée à cette Haute Cour constitutionnelles.

3.Botiveau, op. cit.

4.Une récente pleine page d'Al Ahram Weekly traduisait de manière aiguë les ambiguïtés qui habitent la personne du censeur au ministère de la Culture [à l'époque, cAlî Abû Shâdî], pris en tenaille entre l'héritage que l'on sait et le référent éclairé : cf. Al Ahram weekly, 15-21 juillet 1999, " cAlî Abû Shâdî, the Blade and the Ink », portrait signé par Nadia Abu al-Magd.

5.Voir les «affaires» Youssef Chahine, Nasr Abû Zayd mais aussi le procès contre l'affiche d'un film vieux de vingt ans, Abîfawq al-shagara! Affaires qui ont abouti à ce que la hisba, un précepte de figh reconnu par le droit égyptien, soit effectivement codifiée dans ce dernier - loi nº 3 de 1996 -, dans un sens extrêmement restrictif, il est vrai.

6.Botiveau, op. cit.

7.David I. Kertzer, Ritual, Politics and Power, New Haven and London, YUP, 1988.

8.Sur cette thématique en général, il existe bien sûr une vaste littérature dont on mentionnera ici, pour la perspective linguistique, le célèbre Oswald Ducrot, Dire et ne pas dire, Paris, Hermann, 1972 et, plus récemment, et dans une perspective historique, Arlette Farge, Dire et mal dire, L'opinion publique au XVIII' siècle, Paris, Seuil, coll.

«Librairie du XX ${ }^{\mathrm{e}}$ siècle », 1992.

9.Voir Peter Berger et Thomas Luckman, La construction de la réalité sociale, Paris, Armand Colin, 1996 (1966).

10.Austin, How To Do Things With Words, Oxford, Clarendon Press, 1975.

11.Sharrock et Watson, "L'unité du faire et du dire ", Raisons Pratiques, n 1, 1990.

12.Voir notamment : Michel de Certeau, L'invention du quotidien, (2 vol.), 1. Arts de faire, Paris, Gallimard, coll. « Folio/Essais », 1990 ; et La culture au pluriel, Paris, Christian Bourgois, coll. « Points/Essais », 1993 (1974).

13.Léo Strauss, La persécution et l'art d'écrire, Paris, Agora/Presses Pocket, 1989 (1952).

14.Ch. S. Taylor, « Listening Between the Lines : Some Preliminary Observations on the Construction of Moral Imagination in Late Medieval Egypt », conférence donnée à l'ARCE (Le Caire) en juin 1999.

15.Ferdinand de Saussure, Cours de linguistique générale, Paris, Payot, 1953 (1916).

16. Noam Chomsky, Language and Responsibility: Based on Conversations with Mitsou Ronat, (Transl. from the French by J. Viertel), Sussex, Harvester Press, 1979.

17.Le réinvestissement récent du paradigme nassérien de " projet national » (mashrû qawmî), qui a donné lieu à une quarantaine d'articles, témoigne de ce qu'un topos à l'em-preinte idéologique marquée peut devenir une tribune où se discutent jusqu'à ses fondements même. Sayyid Yasîn, qui a synthétisé le débat par le constat que l'on ne peut parler d'un projet national en termes fonctionalistes (réformer l'administration, réformer l'ensei-gnement, fonder un État laïque en Palestine), propose une refondation du paradigme en terme de rêve national doté de moyens et affecté d'objectifs précis. 18. Howard S. Becker, Outsiders, Études de sociologie de la déviance, Paris, Métailié, 1985. 19.Le Parti du Travail est gelé et son organe al-Shacb suspendu le 20 mai 2000. À cette date, l'ensemble des articles de cette livraison étaient déjà parvenus à la rédaction, ce qui explique qu'ils ne fassent pas mention de cette décision historique. 
INDEX

Mots-clés : censure 